\title{
Suberoylanilide hydroxamic acid, an inhibitor of histone deacetylase, suppresses vasculogenic mimicry and proliferation of highly aggressive pancreatic cancer PaTu8988 cells
}

Xing-dong $\mathrm{Xu}^{1 \dagger}$, Lan Yang ${ }^{1 \dagger}$, Li-yun Zheng ${ }^{2}$, Yan-yan Pan ${ }^{3}$, Zhi-fei Cao ${ }^{3}$, Zhi-qing Zhang ${ }^{4}$, Quan-sheng Zhou ${ }^{3}$, Bo Yang ${ }^{1 *}$ and Cong Cao ${ }^{4^{*}}$

\begin{abstract}
Background: Pancreatic cancer is one of the most aggressive human malignancies with a extremely low 5-year survival rate. Hence, the search for more effective anti-pancreatic cancer agents is urgent.

Methods: PaTu8988 pancreatic cancer cells were treated with different concentrations of suberoylanilide hydroxamic acid (SAHA), cell survival, proliferation, migration and vasculogenic mimicry (VM) were analyzed. Associated signaling changes were also analyzed by RT-PCR and Western blots.

Results: Here, we reported that SAHA, a histone deacetylase inhibitor (HDACi), exerted significant inhibitory efficiency against pancreatic cancer cell survival, proliferation, migration and VM. SAHA dose-dependently inhibited PaTu8988 pancreatic cancer cell growth with the IC-50 of $3.4 \pm 0.7 \mu \mathrm{M}$. Meanwhile, SAHA suppressed PaTu8988 cell cycle progression through inducing G2/M arrest, which was associated with cyclin-dependent kinase 1 (CDK-1)/ cyclin-B1 degradation and p21/p27 upregulation. Further, SAHA induced both apoptotic and non-apoptotic death of PaTu8988 cells. Significantly, SAHA suppressed PaTu8988 cell in vitro migration and cell-dominant tube formation or VM, which was accompanied by semaphorin-4D (Sema-4D) and integrin- $\beta 5$ down-regulation. Our evidences showed that Akt activation might be important for Sema-4D expression in PaTu8988 cells, and SAHA-induced Sema-4D down-regulation might be associated with Akt inhibition.
\end{abstract}

Conclusions: This study is among the first to report the VM formation in cultured human pancreatic cancer cells. And we provided strong evidence to suggest that SAHA executes significant anti-VM efficiency in the progressive pancreatic cancer cells. Thus, SAHA could be further investigated as a promising anti-pancreatic cancer agent.

Keywords: Pancreatic cancer, SAHA, Vasculogenic mimicry, Proliferation and apoptosis

\footnotetext{
*Correspondence: dryangbosz@163.com; caocong@suda.edu.cn

${ }^{\dagger}$ Equal contributors

'Department of General Surgery, the Third Hospital affiliated to Soochow University, Changzhou City 213003, Jiangsu, China

${ }^{4}$ Jiangsu Key Laboratory of Translational Research and Therapy for

Neuro-Psycho-Diseases and Institute of Neuroscience, Soochow University,

Suzhou 215021, Jiangsu, China

Full list of author information is available at the end of the article
} 


\section{Background}

Pancreatic cancer is one of the most aggressive human malignancies, with less than $5 \%$ of patients still alive five years after diagnosis [1]. In 2012, it is estimated that a total of 43,920 patients will be diagnosed with pancreatic cancer in the United States, and 37,390 will die of this disease [2]. Pancreatic cancer is characterized by a rapid disease progression and highly invasive phenotype. Most patients are with unresectable tumor at the time of diagnosis, leaving chemotherapy and radiation as the only available treatment options [3]. For the past decades, gemcitabine has been the standard treatment for advanced pancreatic cancers, prolonging survival by 5-6 months [4]. However, a large percentage of pancreatic cancers do not respond to gemcitabine, probably due to the high level of intrinsic and acquired chemo-resistances [5].

Angiogenesis is essential for tumor growth and metastasis. Tumor-associated angiogenesis is critical for pancreatic cancer progression [6]. Several modes of vessel formation have been proposed so far: vasculogenesis, angiogenesis, intussusceptions, vascular cooption and vasculogenic mimicry (VM) [7]. VM is the process where fluid-conducting channels were formed by the highly invasive and genetically dysregulated tumor cells [8]. Tumors with high VM abilities are often highly aggressive and associated with poor prognosis [8-10]. VM has been observed in a variety of aggressive tumors including carcinomas, breast cancers, liver cancers, ovarian cancers, prostate cancers, sarcomas, gliomas and melanomas [11,12]. Pancreatic cancer represents one of the most vascularized and angiogenic solid tumors [13]. In the current study, we found that many human pancreatic cancer cells could also form tube like structure (VM) in vitro.

In the current study, we aimed to seek novel and more efficient treatment strategies by targeting angiogenic mimicry in pancreatic cancer cells. Suberoylanilide hydroxamic acid (SAHA) belongs to the histone deacetylases (HDAC) inhibitors (HDACi), which represent a new class of anticancer therapeutics. Studies have confirmed its high efficiency in inhibiting angiogenesis in pre-clinical animal models and early phase clinical trials $[14,15]$. SAHA inhibits the in vitro and in vivo growth of transformed human cancer cells, including prostate, bladder and ovarian tumor cells $[15,16]$. SAHA has been tested in phase I and phase II clinical trials for the treatment of various malignancies, and has demonstrated significant anti-cancer efficiency at well-tolerated doses $[15,16]$. Meanwhile, studies have shown that SAHA exhibits profound inhibitory effects against human pancreatic cancer cells [17]. However, the potential effect of SAHA on VM and proliferation of highly metastasis pancreatic cancer cells is not fully studied. Further, the underlying mechanisms remain inconclusive. In this study, we found that
SAHA inhibits in vitro proliferation, migration and VM in a highly aggressive human pancreatic cancer cells (PaTu8988).

\section{Methods}

\section{Chemical and reagents}

SAHA (Purity $\geq 99 \%$ ) was purchased from Selleck Chemicals (Houston, TX). Matrigel and the anti-Semaphorin-4D (Sema-4D) antibody were obtained from BD Biosciences (San Jose, CA). Trypan blue was purchased from Beyotime Biotechnology (Shanghai, China). Annexin V-FITC apoptosis detection kit was purchased from Biotech Co., Ltd (Nanjing, China). RNase-free DNase I was from Qiagen (Hilder, Germany). RevertAid ${ }^{\text {Ts }}$ First Strand cDNA Synthesis Kit was purchased from Fermentas Life Sciences (Chicago, IL). Taq ${ }^{\mathrm{Ts}}$ DNA Polymerase was from TaKaRa Biotechnology Co., Ltd (Dalian, China). Propidium iodide (PI), monoclonal antibody against $\beta$-actin and gelatin were obtained from Sigma (St. Louis, Mo). The anti-cyclinD1 antibody was obtained from ABGENT (Suzhou, China). Anti-epidermal growth factor receptor (EGFR) and platelet-derived growth factor receptor (PDGFR) antibodies were purchased from Santa Cruz Biotech (Santa Cruz, CA). Akt, p-Akt (Ser 473), p70S6 kinase (S6K1), p-S6K1 (Thr 389), S6, p-S6 (Ser 235/236), mTOR, pmTOR (Thr 289), Ulk1, p-Gsk-3 $\beta$, Ulk1, Erk1/2 and p-Erk1/2 antibodies were purchased from Cell Signaling Tech (Beverly, MA). Primers were synthesized by GENEWIZ, Inc. (Suzhou, China).

\section{Cell culture}

As previously described [18], human pancreatic cancer cell lines Pa'Tu8988, Bxpc-3, Aspc-1, CFPAC-1, PaTu8988, SW1990, Panc-1 as well as normal hypertrophic scar fibroblasts (HSF) were obtained from Chinese Academy of Sciences Cell Bank (Shanghai, China). Cells were cultured in RPMI (HyClone, Shanghai, China) with 10\% heatinactivated fetal bovine serum (FBS), with $100 \mathrm{U} / \mathrm{ml}$ of penicillin $\mathrm{G}$ and $100 \mu \mathrm{g} / \mathrm{ml}$ of streptomycin in a $5 \% \mathrm{CO}_{2}$ incubator at $37^{\circ} \mathrm{C}$. Fresh peripheral blood mononuclear cells (PBMNCs) from three healthy adults were collected and separated by Ficoll-Hipaque density sedimentation as previously reported [18], the cells were then cultured in RPMI 1640 medium supplemented with $10 \%$ heatinactivated FBS, $100 \mathrm{U} / \mathrm{ml}$ penicillin $\mathrm{G}$ and $100 \mu \mathrm{g} / \mathrm{mL}$ streptomycin. The study was approved by the institutional review board of the Third Hospital affiliated to Soochow University and all other authors' institutions, and written informed consent was obtained from all three human participants. All clinical investigations were conducted according to the principles expressed in the Declaration of Helsinki. 


\section{Cell growth assay}

Pancreatic cancer PaTu8988 cell growth was assessed using the trypan blue exclusion test. Cells were seeded in 6-well plates for $24 \mathrm{~h}$, various concentration of SAHA was added, cells were further cultured for additional 48 h. Afterwards, cells were harvested and stained with trypan blue. The unstained ("survival") cells were counted in a Neubauer chamber, and the number was expressed as the percentage change of control group. The IC-50, defined as the drug concentration at which cell growth was inhibited by $50 \%$, was assessed by SPSS 16.0 software. All experiments were repeated at least three times.

\section{Colony formation assay}

PaTu8988 cells treated with SAHA for $48 \mathrm{~h}$ were harvest, a total of $1 \times 10^{3}$ cells per well suspended in $150 \mu \mathrm{L}$ of Mix agar with $1.5 \mathrm{~mL}$ DMEM/10\% FBS were plated in $30 \mathrm{~mm}$ plates overlying a $1 \%$ agar-DMEM/10\% FBS (1:1) bottom layer. After 3 weeks, colonies were photographed at $4 \times$. The remaining survival large colonies (>100 $\mu \mathrm{M}$ in diameter) were manually counted.

\section{Cell cycle assay}

PaTu8988 cells were grown in T75 flasks and treated with indicated dosage of SAHA for $48 \mathrm{~h}$. After the treatment, the cells were fixed with $70 \%$ ethanol overnight at $4^{\circ} \mathrm{C}$, washed with PBS, re-suspended in $500 \mu \mathrm{L}$ PBS with $100 \mu \mathrm{g} / \mathrm{mL}$ RNase and incubated for $30 \mathrm{~min}$ at $37^{\circ} \mathrm{C}$. After that, $2.5 \mu \mathrm{L}$ of PI solution $(10 \mathrm{mg} / \mathrm{mL})$ was added. The DNA contents of PI-stained cells were analyzed using a flow cytometry (Becton Dickinson FACS Calibur).

\section{Cell apoptosis assay}

PaTu8988 cell apoptosis was detected by the Annexin V Apoptosis Detection Kit (Beyotime, Shanghai, China) according to the manufacturer's protocol. Briefly, one million cells with indicated treatments were stained with FITC-Annexin V and PI (Beyotime, Shanghai, China). Both early (annexin $\mathrm{V}^{+} / \mathrm{PI}^{-}$) and late (annexin $\mathrm{V}^{+} / \mathrm{PI}^{+}$) apoptotic cells were sorted by fluorescence-activated cell sorting (FACS) (Becton Dickinson FACS Calibur).

\section{Cell morphologic analysis}

A total of $4 \times 10^{4}$ PaTu8988 cells were seeded on glass cover slips in the six well plate and treated with the indicated concentration of SAHA for $48 \mathrm{~h}$. Cells were fixed and stained with Wright-Giemsa stain. The slides were photographed using oil microscopy $(\times 1000$ magnification).

\section{In vitro tube formation assay or vasculogenic mimicry (VM) assay}

The tumor cell formation of capillary structure in vitro was tested as we previously described $[19,20]$.

\section{Cellular immuno-fluorescence staining}

PaTu8988 cells were seeded on glass cover slips in six well plates and treated with described dosage of SAHA for $48 \mathrm{~h}$. Cells on the cover slip were then fixed with $4 \%$ paraformaldehyde for $10 \mathrm{~min}$ at room temperature without permeabilization. Slides were washed three times with phosphate buffered saline (PBS), blocked with $5 \%$ bovine serum albumin (BSA) for $1 \mathrm{~h}$ at $37^{\circ} \mathrm{C}$, followed by incubation with the primary antibody overnight at $4^{\circ} \mathrm{C}$, and the secondary antibody for $1 \mathrm{~h}$ at room temperature. The slides were photographed using OLYMPUS FSX100 microscope.

\section{MTT cell viability assay}

The cell viability was measured by the 3-[4,5dimethylthylthiazol-2-yl]-2,5 diphenyltetrazolium bromide (MTT) method, as described before [21]. Briefly, the PaTu8988 cells were collected and seeded in 96-well plate at a density of $2 \times 10^{5}$ cells $/ \mathrm{cm}^{2}$. Different seeding densities were optimized at the beginning of the experiments. After treatment, $20 \mu \mathrm{l}$ of MTT tetrazolium (Sigma, St. Louis, MO) salt dissolved in PBS at a concentration of $5 \mathrm{mg} / \mathrm{mL}$ was added to each well and incubated in a $\mathrm{CO}_{2}$ incubator for additional 2 hrs. Finally, the medium was aspirated very carefully and $150 \mu \mathrm{l} /$ well of DMSO (Sigma, St. Louis, MO) was added to dissolve formazan crystals. The absorbance of each well was obtained using a plate reader at a test wavelength of $490 \mathrm{~nm}$ with a reference wavelength of $630 \mathrm{~nm}$. The value of treatment group was always normalized to that of control group.

\section{"Scratch" assay}

As described [22], twelve-well plates were pre-coated with poly-lysine $(30 \mu \mathrm{g} / \mathrm{ml})$, followed by further BSA blocking. A sufficient number of PaTu8988 cells were plated, so that they became confluent in the wells right after attachment $(\sim 1-2 \mathrm{~h})$. Same area of each well is then displaced by scratching a same straight line through the layer with a needle. Floating cells were washed away by warm PBS. Cells were further incubated with the indicated concentration of SAHA for $24 \mathrm{~h}$, and stained with Wright-Giemsa to see migration "gap". Mitomycin C $(10 \mu \mathrm{g} / \mathrm{ml})$ was always included in the culture media to prevent cell proliferation.

\section{PCR analysis}

Total RNA was extracted from PaTu8988 cells and treated with RNase-free DNase I. The quality of RNA was test by DU-800 Nucleic Acid/Protein Analyzer (Beckman, 
U.S.A). The cDNA was generated by reverse transcription using RevertAidTM First Strand cDNA Synthesis Kit and oligo $(\mathrm{dT})$ in a $20 \mu \mathrm{L}$ reaction containing $5 \mu \mathrm{g}$ of total RNA. Next, PCR was performed in each $25 \mu \mathrm{L}$ PCR reaction containing $0.5 \mu \mathrm{L}$ diluted cDNA, TaKaRa rTaq DNA Polymerase and indicated primers. The PCR reaction contained an initial denaturation at $94^{\circ} \mathrm{C}$ for
3 min, followed each PCR cycle by de-naturation at $94^{\circ} \mathrm{C}$ for 30 seconds, annealing at $55-68^{\circ} \mathrm{C}$ for 30 seconds, and extension at $72^{\circ} \mathrm{C}$ for $1 \mathrm{~min}$ for a total of 22-36 cycles, depending on the primer length and the molecular weights of target genes. PCR products were analyzed by $1.5 \%$ agarose gel. Primers used in this study were summarized in Table 1.

Table 1 Primer sequences for semi-quantitative RT-PCR

\begin{tabular}{|c|c|c|c|}
\hline Gene name (mRNA ID) & Primer name & Primers sequences $\left(5^{\prime}-3^{\prime}\right)$ & PCR product size (bp) \\
\hline$\beta$-actin & Forw & AAGAGCTACGAGCTGCCTGACG & \multirow{2}{*}{420} \\
\hline (NM_001101.3) & Rev & CGCCTAGAAGCATTTGCGGTGG & \\
\hline Cyclin D1 & Forw & CATCTCTGTACTTTGCTTGCTCAT & \multirow{2}{*}{499} \\
\hline (NM_053056.2) & Rev & CGCTATTTCCTACACCTATTGGAC & \\
\hline Cyclin B1 & Forw & TCAACATGGCAGGCGCAAAGC & \multirow{2}{*}{224} \\
\hline (NM_031966.3) & Rev & TGGCACTGGCACCAGCATAGGT & \\
\hline CDK1 & Forw & TGCTGGGGTCAGCTCGTTACTCA & \multirow{2}{*}{232} \\
\hline (NM_001786.4) & Rev & TGGGATGCTAGGCTTCCTGGTT & \\
\hline p53 & Forw & GGGAGTAGGACATACCAGCTTAGAT & \multirow{2}{*}{452} \\
\hline (NM_000546.4) & Rev & TTAGGTACTAAGGTTCACCAAGAGG & \\
\hline p21 & Forw & CTGCCTTAGTCTCAGTTTGTGTGT & \multirow{2}{*}{412} \\
\hline (NM_000389.3) & Rev & CAAAGTGCCATCTGTTTACTTCTC & \\
\hline CDK4 & Forw & CTTGATCTGAGAATGGCTACCTCT & \multirow{2}{*}{409} \\
\hline (NM_000075.2) & Rev & CATGAAGGAAATCTAGGCCTCTTA & \\
\hline MAGEF1 & Forw & GGGTATCCGAAGAGGCTTATTATGG & \multirow{2}{*}{749} \\
\hline (NM_022149.4) & Rev & AAGCAAATGAAGGTACATGCCAGTC & \\
\hline FGFR1 & Forw & GAGGAGAAAGAAACAGATAACACCA & \multirow{2}{*}{427} \\
\hline (NM_023108.2) & Rev & TGTACACCTTACACATGAACTCCAC & \\
\hline HIF1A & Forw & GTAAGAAGGCAGTAACCTTTCATCA & \multirow{2}{*}{502} \\
\hline (NM_001530.3) & Rev & AGGGTAGGCAGAACATTTAGGTTAA & \\
\hline RUNX1 & Forw & AGATTTAATGACCTCAGGTTTGTCG & \multirow{2}{*}{328} \\
\hline (NM_001754.4) & Rev & GACTCTGAGGCTGAGGGTTAAAG & \\
\hline Sema4D & Forw & TGTCTGTGGAGTATGAGTTGTGTT & \multirow{2}{*}{552} \\
\hline (NM_006378.3) & Rev & GGGTGTAGTTCACATCTTTCTTGAT & \\
\hline VEGFA & Forw & AGAAGAGACACATTGTTGGAAGAAG & \multirow{2}{*}{439} \\
\hline (NM_003376.4) & Rev & CGGTACAAATAAGAGAGCAAGAGAG & \\
\hline MAGED1 & Forw & TCGGTCTCCTCTTGGTGATTCTGG & \multirow{2}{*}{200} \\
\hline (NM_006986.3) & Rev & GTTGCTGTTGGGCACTCGTCTGT & \\
\hline Intergrin $\beta 5$ & Forw & TGCCATGCAGGTTACATCGG & \multirow{2}{*}{353} \\
\hline (NM_002213) & Rev & ATCATGACGCAGTCCTTGGC & \\
\hline WIPF2 & Forw & СTCCTITACTCAGACATAGAGCATCA & \multirow{2}{*}{484} \\
\hline (NM_133264.4) & Rev & AGACCACCCAATCTTCCACGAA & \\
\hline ZFP106 & Forw & СССАССТСТСАССССТАТTТССТА & \multirow[t]{2}{*}{280} \\
\hline (NM_022473.1) & Rev & AACACCTCACCTACCCGTTTCCTG & \\
\hline
\end{tabular}

Note: Forw: the forward primer; Rev: the reverse primer; CDK1: cyclin-dependent kinase 1; CDK4: cyclin-dependent kinase 4; FGFR1: fibroblast growth factor receptor 1; HIF1A: hypoxia inducible factor 1, alpha subunit; MAGED1: melanoma antigen family D1; FGFR1:fibroblast growth factor receptor 1; VEGFA: vascular endothelial growth factor A; Sema4D:Sema domain, immunoglobulin domain (Ig), transmembrane domain and short cytoplasmic domain, 4D; WIPF2: WAS/WASL interacting protein family, member 2; MAGEF1: melanoma antigen family F1; MAGED1: melanoma antigen family D1; ZFP106: zinc finger protein 106 homolog; RUNX1: runt-related transcription factor 1. 


\section{Western blot analysis}

As described before [21], aliquots of 30-40 $\mu \mathrm{g}$ of protein from each sample (treated as indicated in the legends) was separated by $10 \%$ SDS-polyacrylamide gel electrophoresis (SDS-PAGE) and transferred onto a polyvinylidene difluoride (PVDF) membrane (Millipore, Bedford, MA). After blocking with $10 \%$ instant nonfat dry milk for $1 \mathrm{~h}$, membranes were incubated with the specific antibody overnight at $4^{\circ} \mathrm{C}$, followed by incubation with corresponding secondary antibody (HRP-conjugated antirabbit or anti-mouse IgG at the appropriate dilutions) for $30 \mathrm{~min}$ to $1 \mathrm{~h}$ at room temperature. Antibody binding was detected with the enhanced chemiluminescence (ECL) detection system (Amersham Biosciences, Piscataway, NJ). The intensity of interested band was quantified using ImageJ software, and the value was normalized to corresponding loading controls.

\section{Statistic analysis}

The data shown in this study represented the mean \pm S.E. Differences between the groups were assessed by one-way ANOVA using SPSS 16.0 software. The significance of differences was indicated as ${ }^{*} P<0.05$ and ${ }^{* *} P<0.01$.

\section{Results}

SAHA inhibits the proliferation of PaTu8988 pancreatic cancer cells

Figure 1A showed the chemical structure of SAHA. Considering that uncontrolled proliferation and robust angiogenesis (i.e. VM) contribute to the growth and metastasis of pancreatic cancers, we first investigated the potential role of SAHA on the pancreatic cancer cell proliferation. As shown in Figure 1B, SAHA dosedependently inhibited PaTu8988 cell proliferation with the IC-50 of $3.4 \pm 0.7 \mu \mathrm{M}$. However, it had almost no effect on the proliferation of HSF (hypertrophic scar fibroblasts) (Figure 1E) and normal PBMNCs (peripheral blood mononuclear cells) (Figure 1F) at the dose up to $40 \mu \mathrm{M}$. These results suggested that SAHA has selective inhibitory efficiency against pancreatic cancer cells, but not normal mononuclear cells or HSF cells. To further explore the inhibitory ability of SAHA on PaTu8988 cell proliferation under more stringent conditions, the colonial survival assay was performed. The results showed that the number of remaining survival colonies in SAHAtreated group was significantly lower than that of control group (Figure $1 \mathrm{C}$ and D). Hence, these results demonstrated that SAHA effectively inhibits PaTu8988 cell in vitro proliferation.

\section{SAHA affects cell cycle progression of PaTu8988 cells}

Next, we analyzed the cell cycle distribution in SAHAtreated PaTu8988 cells. As shown in Figure 2A and B, a large population of SAHA $(>5-10 \mu \mathrm{M})$-treated PaTu8988 cells were arrested in G2/M phase. Meanwhile, RT-PCR results showed that the mRNA expressions of cyclindependent kinase 1 (CDK-1), cyclin-D1 and cyclin-B1 were down-regulated after SAHA treatment, while the p21 and p27 mRNAs were markedly increased (Figure 2C). The CDK-2, CDK-4 and p53 mRNAs were not affected by SAHA (Figure $2 \mathrm{C}$ ). Further, western blot results in Figure 2D confirmed that the protein level of cyclin-D1 was markedly decreased after SAHA $(10$ or $20 \mu \mathrm{M})$ treatment, while p21 and p27 protein expressions were significantly upregulated. Immuno-fluorescence results in Figure $2 \mathrm{E}$ further confirmed p21 upregulation and nuclear translocation after SAHA stimulation in PaTu8988 cells. These results suggested that SAHA suppresses cell cycle progression by inducing G2/M arrest in PaTu8988 cells; such effect of SAHA is associated with perturbation of cell cycle-associated proteins.

\section{SAHA induces both apoptotic and non-apoptotic death of PaTu8988 cells}

Next, we examined whether the inhibitory effect of SAHA on PaTu8988 cell proliferation was due to cell apoptosis. As shown in Figure 3A and B, the population of apoptotic (Annexin V positive) PaTu8988 cells increased significantly after high dose SAHA $(>10 \mu \mathrm{M})$ treatment. Meanwhile apoptosis-associated proteins were also changed. Poly (ADP-ribose) polymerase (PARP) and caspase-3 were down-regulated after SAHA treatment, while cleaved-PARP was up-regulated (Figure $3 \mathrm{C}$ ). We failed to see an increase of cleaved-caspase- 3 in SAHAtreated PaTu8988 cells (Figure 3C). Interestingly, we also noticed a small population of non-apoptotic "dead" PaTu8988 cells (Annexin V negative and PI positive) after SAHA treatment. Together, these results suggested that both apoptotic and non-apoptotic cell death might contribute to SAHA-induced anti-proliferation effect in PaTu8988 cells.

\section{SAHA induces differentiation and inhibits migration of PaTu8988 cells}

We also examined the potential effect of SAHA on the morphology change of PaTu8988 cells. The PaTu8988 cells were incubated with SAHA for $48 \mathrm{~h}$. Afterwards, cells were stained with Wright-Giemsa to see their morphology. As shown in Figure 4A, control cells were small and had little hyper-chromatism in cytoplasm, indicating an undifferentiated shape. While the SAHA-treated cells were bigger, and were with full of light cytoplasm and cytoplasm projections: a typical differentiated shape. These results suggested that SAHA might induce PaTu8988 cell differentiation. We also tested the effect of SAHA on cell migration through in vitro "scratch" assay; results in Figure $4 \mathrm{~B}$ demonstrated that SAHA dose-dependently 
A<smiles>O=C(CCCCCCC(=O)Nc1ccccc1)NO</smiles>

Vorinostat (SAHA)

C

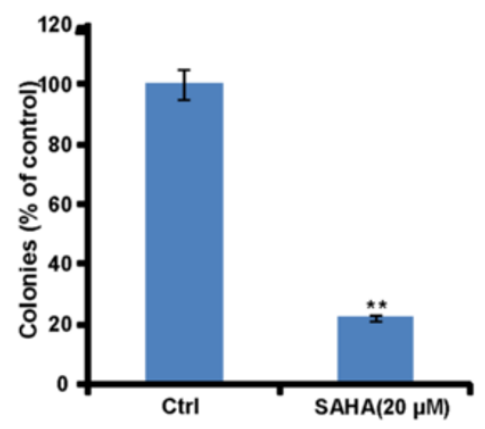

E
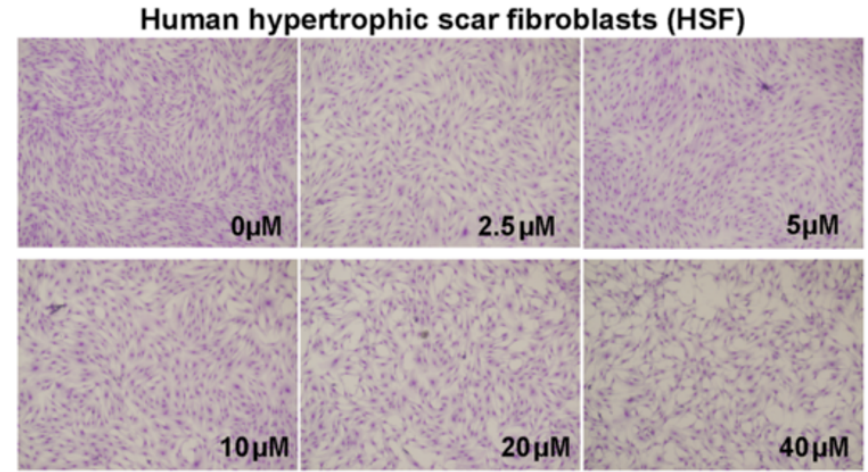

$\mathbf{F}$

F Peripheral blood mononuclear cells (PBMNCs)

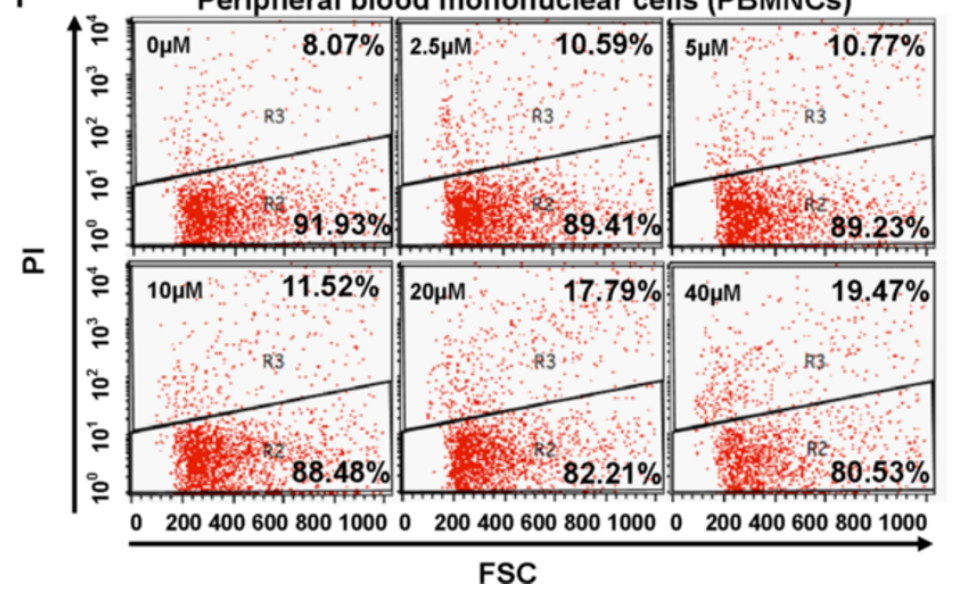

FSC

Figure 1 SAHA inhibits the proliferation of PaTu8988 pancreatic cancer cells. Chemical structure of SAHA is shown in (A). Human pancreatic cancer PaTu8988 cells were incubated with SAHA at indicated dosage for $48 \mathrm{~h}$, and cell growth was measured by trypan blue exclusion test. The IC-50 was calculated by SPSS 16.0 software (B). PaTu8988 cells were treated with SAHA (20 MM). The colony formation assay was performed, and the number of colonies was manually counted (C and D). Human hypertrophic scar fibroblasts (HSF) were treated with various concentration of SAHA for $48 \mathrm{~h}$, followed by Wright-Giemsa staining and photograph by OLYMPUS FSX-100 microscope (E). Peripheral blood mononuclear cells (PBMNCS) were treated with various concentration of SAHA for $48 \mathrm{~h}$, cell death was detected by PI staining through FACS sorting (F). Experiments in this figure were repeated three times, and similar results were obtained. The data in this figure was expressed as mean \pm S.E. ${ }^{* *} P<0.01$ vS. Ctrl (C). Magnification: 1:100 (E). 

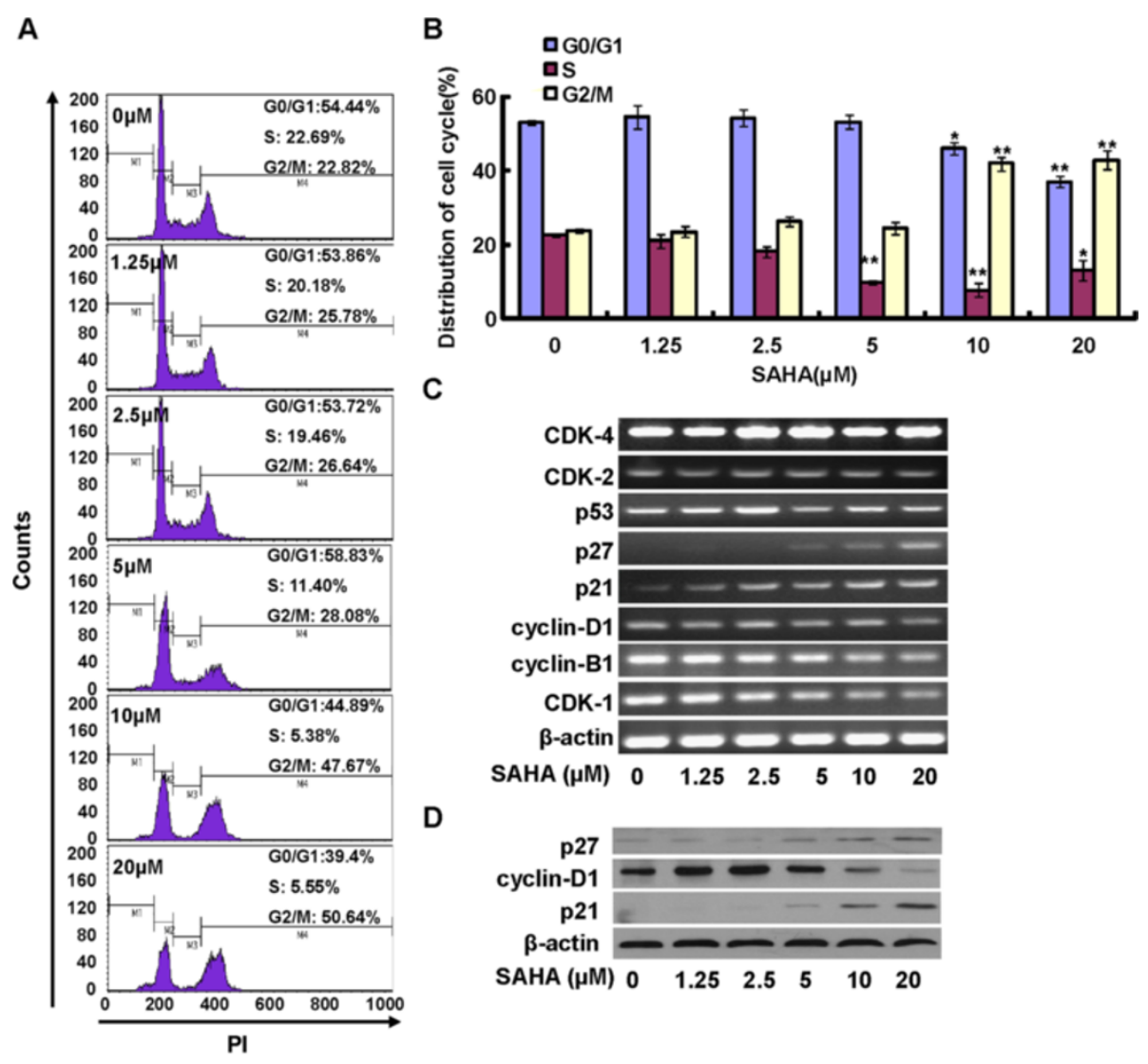

C

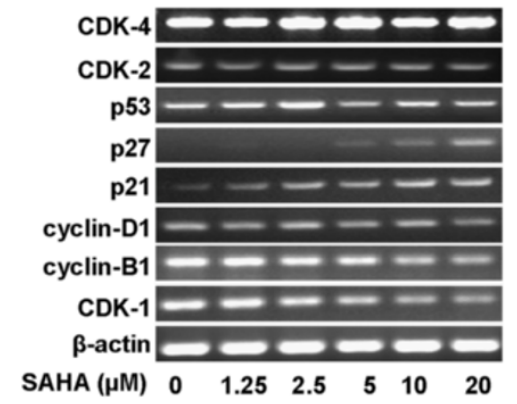

D

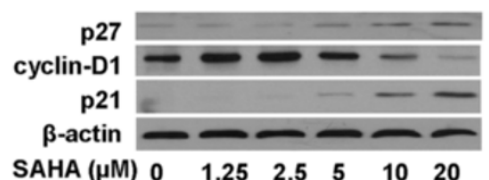

E
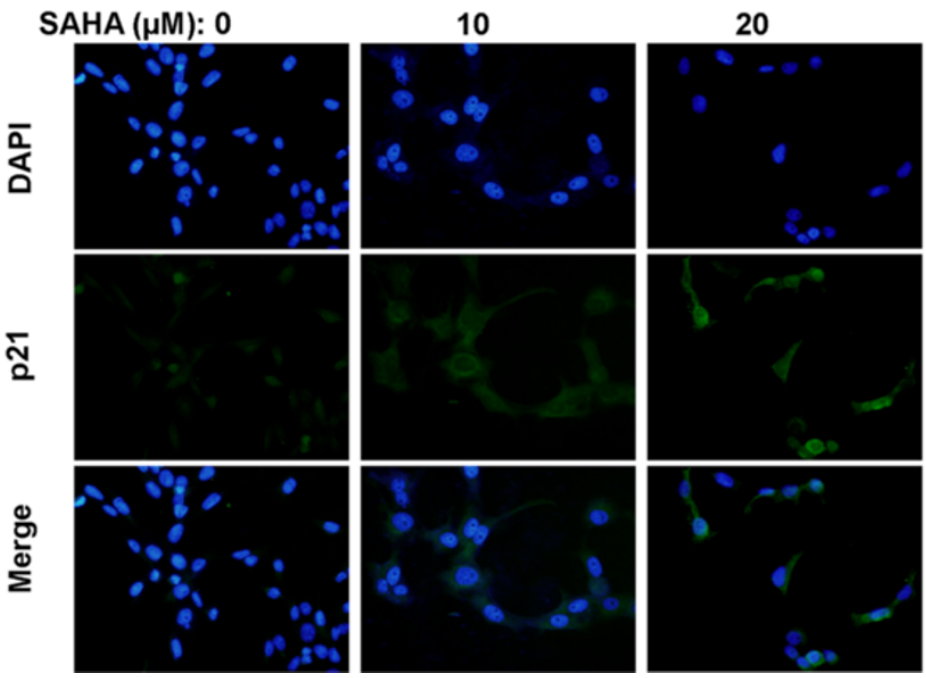

Figure 2 SAHA affects cell cycle progression of PaTu8988 cells. PaTu8988 cells were incubated with SAHA at indicated dosage for 48 h, DNA content of Pl-stained cells was analyzed by flow cytometry (A), and cell cycle distribution was analyzed (B). The mRNAs of cell cycle regulatory genes were detected by semi-quantitative RT-PCR assay (C). The protein expressions of cyclin-D1, p21, p27 and $\beta$-actin were also tested by western blots (D), and the expression and sub-cellular location of p21 were also measured by immuno-fluorescence (E). Experiments in this figure were repeated three times, and similar results were obtained. The data in this figure was expressed as mean \pm S.E. ${ }^{*} P<0.05$ vs. Ctrl, ${ }^{* *} P<0.01$ vs. Ctrl (B). Magnification: 1:400 (E).

suppressed the "gap" closing, indicating its inhibitory efficiency against PaTu8988 cell in vitro migration. The inhibitory effects of SAHA on cell migration were not secondary to decreased viability, as no significant cell viability decrease was observed after indicated SAHA treatment for $24 \mathrm{~h}$ (Figure 4C). 

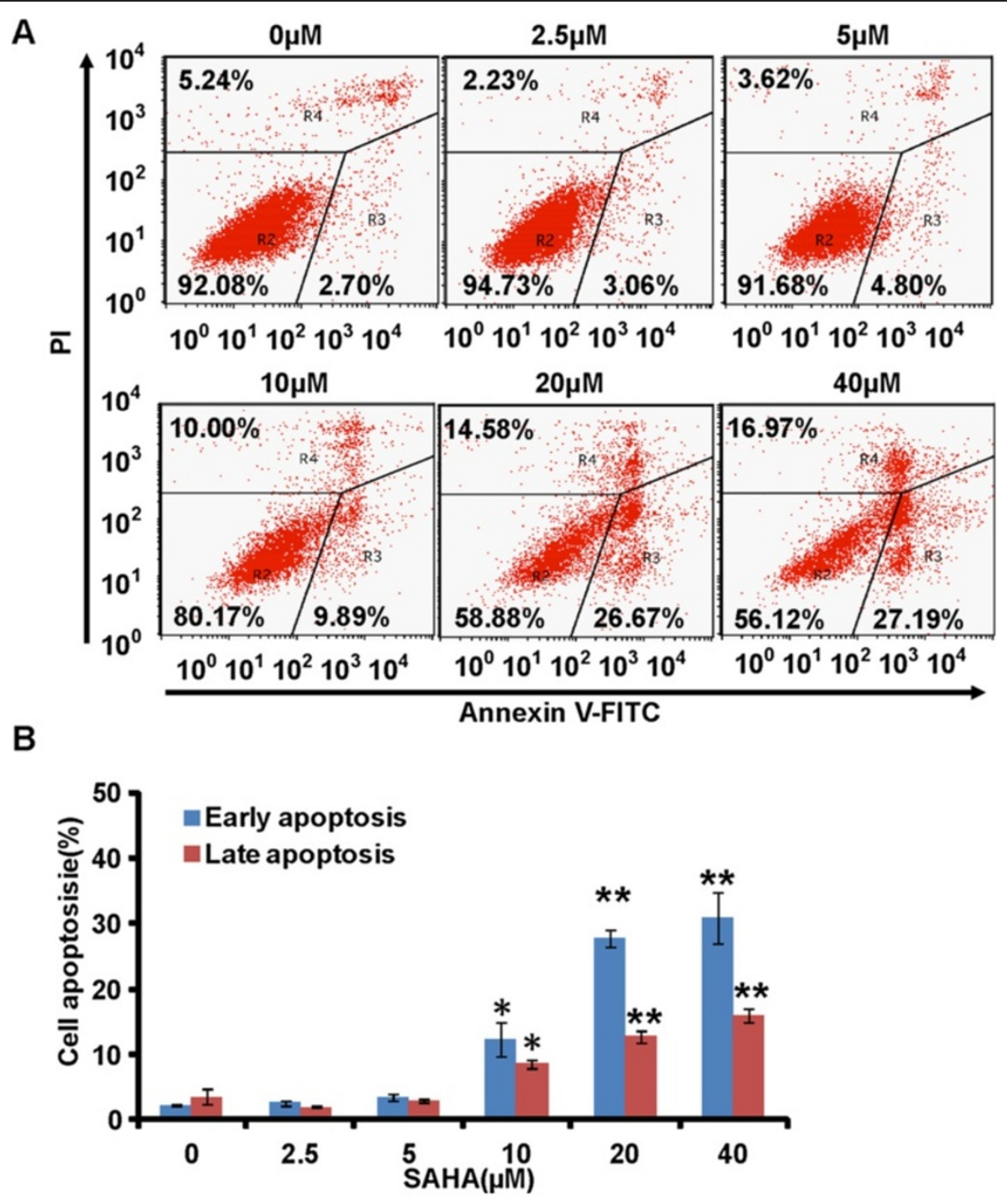

C

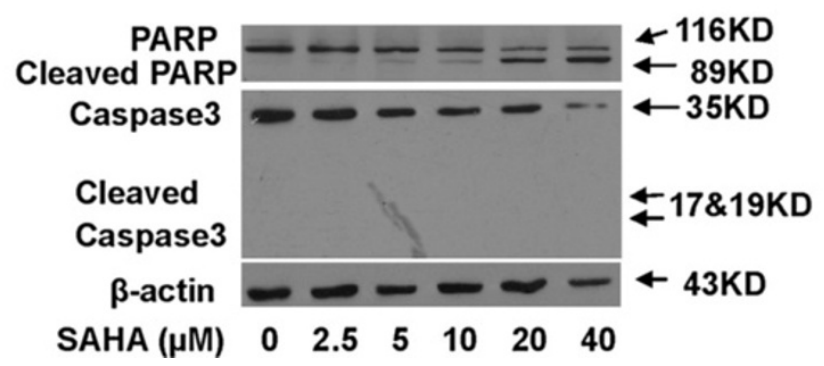

Figure 3 SAHA induces both apoptotic and non-apoptotic death of PaTu8988 cells. PaTu8988 cells were incubated with SAHA at indicated dosage for $48 \mathrm{~h}$, FITC-annexin V and PI stained cells were sorted by flow cytometry (A). The distribution of cell apoptosis was analyzed (B). The protein expressions of PARP, cleaved-PARP, caspase-3, cleaved-caspase-3 and $\beta$-actin were detected by western blots (C). Experiments in this figure were repeated three times, and similar results were obtained. The data in this figure was expressed as mean \pm S.E. ${ }^{*} P<0.05$ vs. Ctrl, ${ }^{* *} P<0.01$ vs. Ctrl (B).

SAHA suppresses PaTu8988 cell vasculogenic mimicry (VM) Results above have shown that SAHA inhibits PaTu8988 cell in vitro migration. VM is the formation of fluid- conducting channels by highly invasive and genetically dysregulated tumor cells [9]. Through in vitro tube formation assay, we observed the VM formation in multiple 


\section{A}

SAHA $(\mu M): 0$

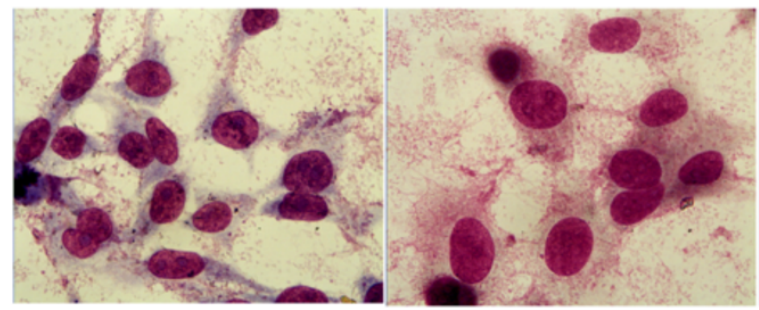

2.5

10

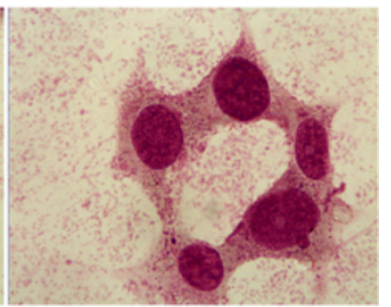

B

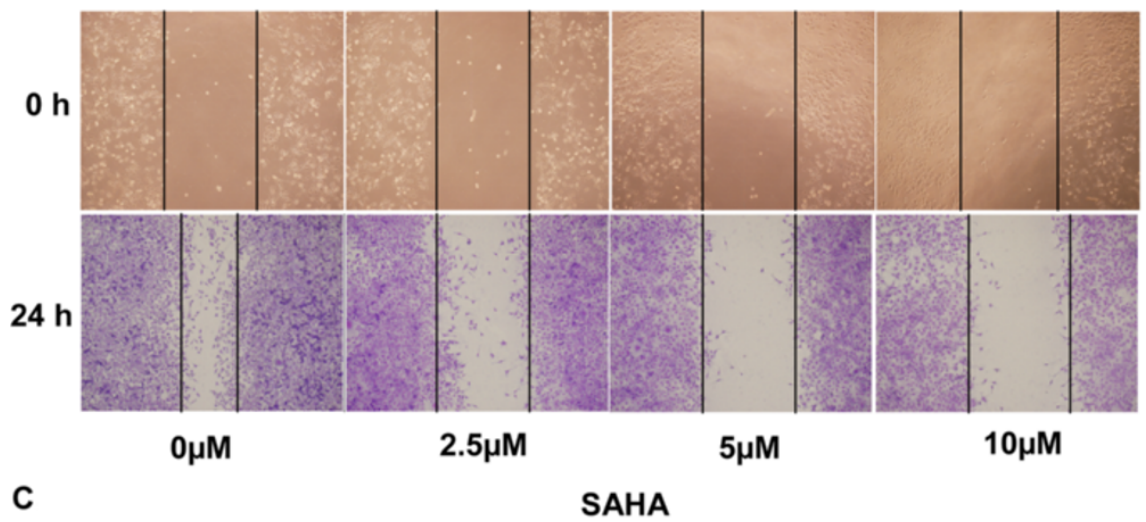

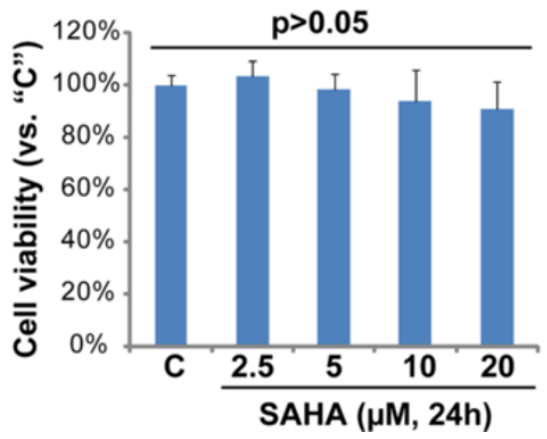

Figure 4 SAHA induces differentiation and inhibits migration of PaTu8988 cells. (A) PaTu8988 cells were seeded on glass cover slips in six-well plate and treated with SAHA for $48 \mathrm{~h}$. Cells were stained with Wright-Giemsa stain and photographed by oil microscopy for 1000X. (B) PaTu8988 cells were incubated with SAHA at indicated dosage for $24 \mathrm{~h}$, the in vitro cell migration was analyzed by "scratch" assay as described, cell viability was analyzed by MTT assay (C). Experiments in this figure were repeated three times, and similar results were obtained. Magnification: 1:200 (B).

human pancreatic cancer cells (Bxpc-3, PaTu8988, Panc-1 and CFPAC-1) (Figure 5A). To examine whether SAHA have anti-VM ability, the PaTu8988 cells, pretreated with or without SAHA, were seeded onto a Matrigel layer and the capillary tube formation ability was monitored and photographed. As shown in Figure 5B-C, the PaTu8988 cells again formed a good tube like structure, which was inhibited by SAHA. Note that $20 \mu \mathrm{M}$ of SAHA almost completely disrupted VM formation. VM-associated genes were also tested in control and SAHA-treated PaTu8988 cells. As shown in Figure 5D, Sema-4D and integrin- $\beta 5$ mRNAs were significantly down-regulated by SAHA (10 and $20 \mu \mathrm{M}$ ), and the HIF-2A mRNA expression was also suppressed by SAHA $(20 \mu \mathrm{M})$. Interestingly, other tumor VM and angiogenic genes including RUNX1, HIF (Hypoxia-inducible factor)-1A, integrin- $\alpha 5$ and VEGF (vascular endothelial growth factor)-A were not affected. Further, western blot results (Figure 5E) confirmed that Sema-4D protein was down-regulated by SAHA in PaTu8988 cells. Hence, these results suggested that SAHA inhibited PaTu8988 cell in-vitro VM, which was associated with Sema-4D and integrin- $\beta 5$ down-regulation.

\section{Akt is important for Sema-4D expression in PaTu8988 cells, inhibited by SAHA}

Since previous studies have confirmed that Akt and its downstream mTORC1 is important for both survival and migration of pancreatic cancer cells [23-29], we thus 
A

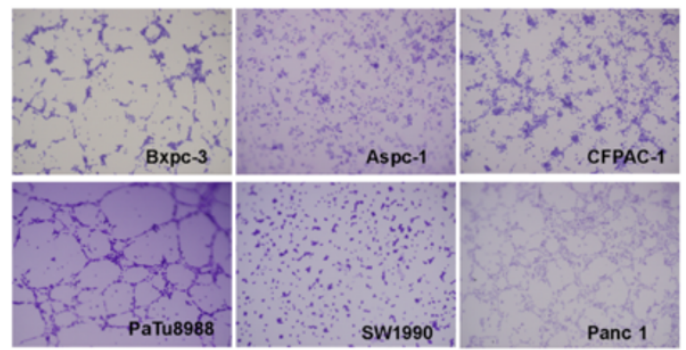

B

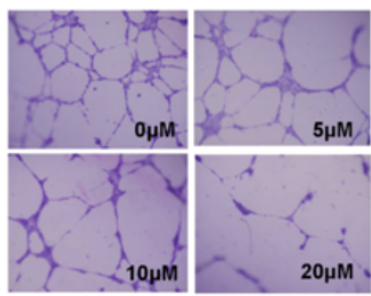

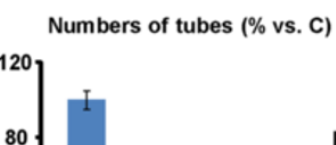

D

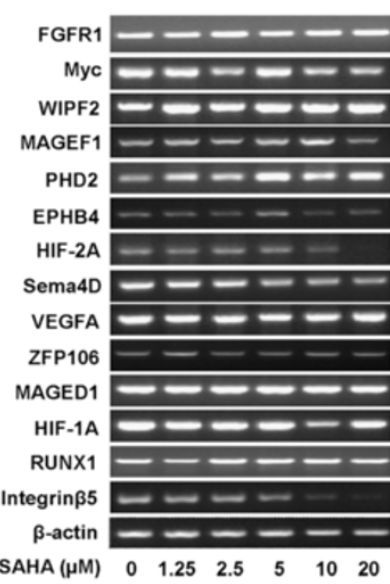

E

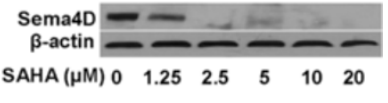
* SAH

Figure 5 SAHA suppresses PaTu8988 cell vasculogenic mimicry (VM). The six human pancreatic cancer cells including Bxpc-3, Aspc-1, CFPAC-1, PaTu8988, SW1990 and Panc-1 were harvested and suspended in RPMI-1640 plus 10\% FBS at a density of $2 \times 10^{5} / \mathrm{mL}$. A volume of $150 \mu \mathrm{L} /$ well of Matrigel matrix was transferred to a 48 -well plate at $37^{\circ} \mathrm{C}$ for $30 \mathrm{~min}$. Then the cells were transferred to each well. The cells were further incubated at $37^{\circ} \mathrm{C}, 5 \% \mathrm{CO}_{2}$ for $4-6 \mathrm{~h}$, the formed tubes were stained with Wright-Giemsa and photographed by OLYMPUS FSX-100 microscope (A). PaTu8988 cells were treated with the indicated concentration of SAHA for $24 \mathrm{~h}$, and subjected to a tube formation assay as described (B). The capillary-like structures were imaged and analyzed for quantification $\mathbf{( C )}$. The mRNAs of vasculogenic and angiogenic genes were determined by semi-quantitative RT-PCR assay (D), and the expression of Sema-4D was detected by western-blot (E). Experiments in this figure were repeated three times, and similar results were obtained. The data in this figure was expressed as mean \pm S.E. ${ }^{* *} P<0.01$ vs. Ctrl (C). Magnification: $1: 200$ (A and B).

wanted to know whether SAHA could affect activation of Akt-mTORC1 in PaTu8988 pancreatic cancer cells. Also, it has been suggested that Akt signaling is linked with cancer cell VM [30,31], we tested whether this signaling pathway was important for Sema-4D expression. As shown in Figure 6A and B, SAHA (10-40 $\mu \mathrm{M})$ significantly inhibited activation of Akt. Meanwhile, mTORC1 activation, indicated by p-mTOR, p-S6K1 and p-S6, was also suppressed by SAHA (Figure 6A and B). Expression of Ulk1, an indicator of autophagy activation, was not affected by SAHA treatment (Figure 6A). We proposed that growth factor receptors' degradation might be responsible for Akt-mTORC1 inhibition by SAHA, since SAHA administration down-regulated epidermal growth factor receptor (EGFR) and platelet-derived growth factor receptor $\beta$ (PDGFR $\beta$ ) expression (Figure 6C). Interestingly, as shown in Figure 6D, the Akt inhibitor perifosine, but not the mTORC1 inhibitor rapamycin, inhibited Sema-4D expression in PaTu8988 cells, indicating that Akt rather than mTORC1 is important for Sema-4D expression. Even more intriguingly, although perifosine blocked Akt activation, it only inhibited, but not blocked S6 phosphorylation. These results suggested that other upstream signals beside Akt might also be responsible for mTORC1 or S6 activation in this particular cell line, and that SAHA's inhibitory ability on mTORC1 activation might not solely depend on Akt inhibition.

\section{Discussion}

Gemcitabine is the only standard chemotherapy for pancreatic cancer patients. However, the median survival with gemcitabine treatment was still a dismal 5.65 months with 1-year survival rate of $18 \%$ [32]. In the current study, we used PaTu8988 pancreatic cancer cells as a cell model to investigate anti-cancer activity of SAHA. Our results demonstrated that SAHA exerted profound inhibitory efficiency against PaTu8988 cells. SAHA dramatically inhibited PaTu8988 cell survival, proliferation, migration, and more importantly tuber formation or VM. This study is among the first to report the VM formation in human pancreatic cancer cells. Further, we provided strong evidence to suggest that SAHA executed a significant anti-VM effect in human pancreatic cancer cells. Meanwhile, SAHA also promoted cancer cell cycle arrest and cell death (both apoptotic and non-apoptotic). Thus, SAHA could be further investigated as a promising antipancreatic cancer agent.

SAHA induces PaTu8988 cell cycle arrest at G2/M phase probably via down-regulating cyclin B1. Previous 


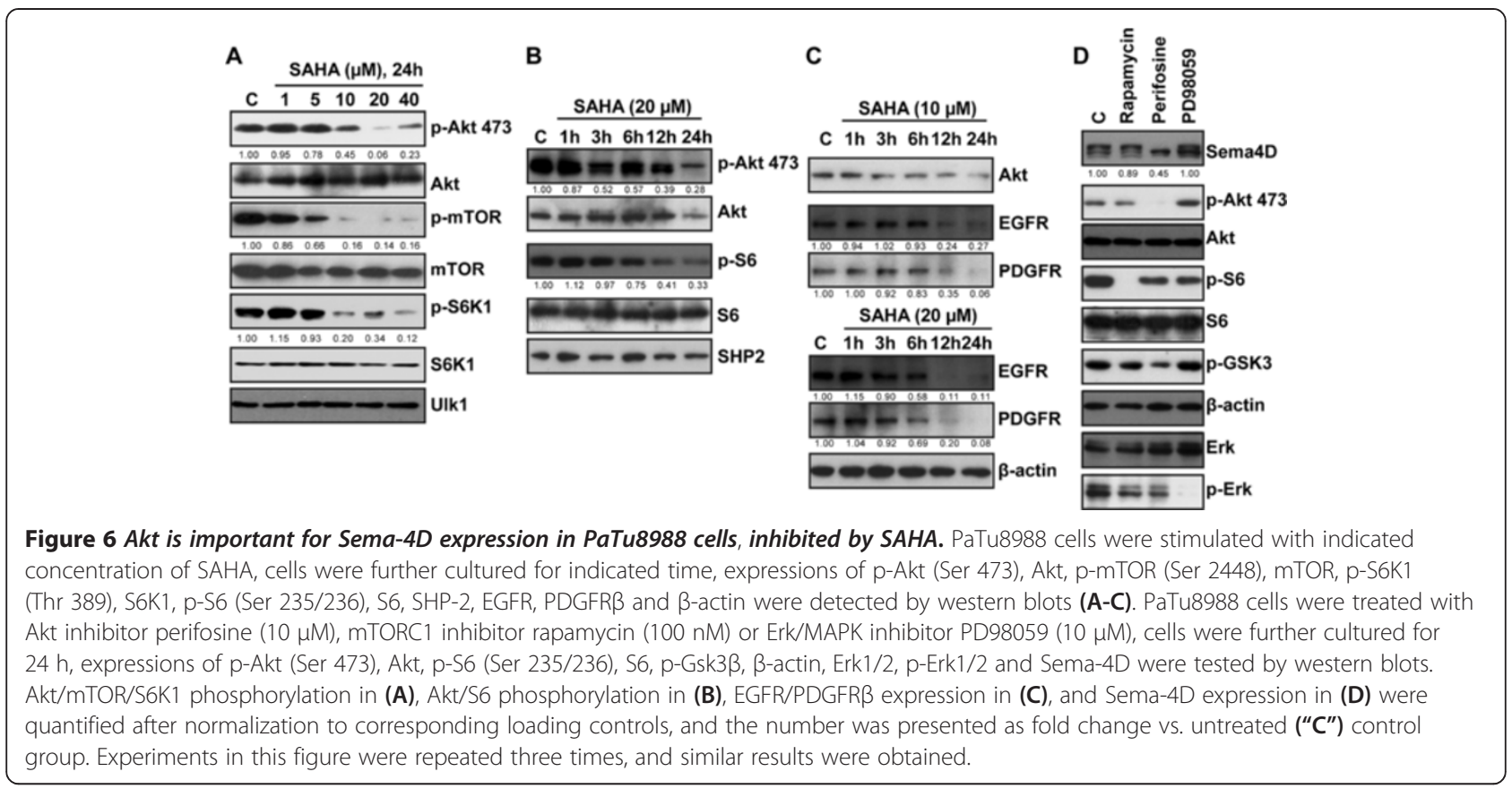

studies have shown that cyclin B1 degradation is actively involved in G2/M arrest [33]. And constitutive activation of cyclin B1 overrides p53-mediated G2/M arrest [32]. In our study, we found that SAHA induced expressions of CDK inhibitors p21 and p27, which are known to affect G2/M cycle progression [34]. Here we observed a significant cell apoptosis after high dose of SAHA treatment, the mechanism of SAHA-induced apoptosis may be associated with PARP and caspase- 3 degradation, as suggested by other studies [34,35]. Intriguingly, SAHA also induced non-apoptotic cell death in PaTu8988 cells. This result is not surprising, as recent studies have observed non-apoptotic death, in particular autophagic cell death induced by SAHA $[36,37]$.

Tumor vasculogenic mimicry (VM), which is characterized by the tumor cell-lined vessels, was first found from metastatic melanoma by Hendrix MJ group in 1999 [34]. Hence, VM has been targeted for anti-cancer therapy. Here we first reported that multiple pancreatic cancer cell lines formed a good tube like structure in Matrigel in vitro. Significantly, SAHA greatly inhibited PaTu8988 cell-mediated VM in vitro, such an effect was associated with down-regulating Sema- $4 \mathrm{D}$ and integrin- $\beta 5$, two key VM associated proteins.

Here we observed a significant down-regulation of Sema-4D by SAHA in PaTu8988 cells. Sema-4D expression is seen in a wide range of human tumors including prostate, colon, breast, oral, head and neck carcinomas [37]. Sema-4D is a cell surface membrane protein that is shed from tumor cells and promotes endothelial cell proliferation, migration, angiogenesis, and tumor invasive growth through its action on its cognate endothelial receptor, plexin B1 [35]. In the absence of Sema-4D, tumor growth and tumor angiogenesis in vivo are greatly impaired [36]. Researchers have demonstrated that Sema-4D can potentiate the invasiveness of pancreatic cancer cells. In the present study, we found that SAHA downregulated Sema-4D expression in PaTu8988 cells, which may be one the mechanism responsible for VM disruption. To our knowledge, this is the first report showing SAHA affects Sema-4D expression and cancer cell VM.

Integrin $\beta 5$ is another potent angiogenic gene whose expression in PaTu8988 cells was also suppressed by SAHA. Integrins are a family of non-covalently associated het-erodimeric cell surface receptors composed of a $\alpha$ and $\beta$ subunit that mediate cell-ECM and cell-cell adhesions [38]. It is reported that mice lack of integrin $\beta 3$ and $\beta 5$ showed less tumorigenesis [39]. We found that PaTu8988 cells treated with SAHA showed inhibited expression of integrin $\beta 5$, another mechanism to explain SAHA's anti-angiogenic potential.

Pancreatic cancers are among the most intrinsically resistant tumors to almost all classes of cytotoxic drugs [38]. The extremely high level of drug resistance was associated with dysregulation of multiple signaling pathways $[39,40]$. One key signaling pathway that is frequently over-activated in pancreatic cancer is Akt/mTOR signaling cascade [39,40], which is responsible for cancer cell survival, proliferation, apoptosis-resistance, migration and metastasis $[39,40]$. The fact that SAHA significantly inhibited Akt and S6 activation in PaTu8988 cells might explain its inhibitory efficiency against this cell line. As a 
matter of fact, our data showed that perifosine, the Akt inhibitor, significantly inhibited PaTu8988 cell proliferation, migration and survival (Data not shown).

Importantly, recent studies have indicated that Akt signaling is also important for cancer cell vasculogenic mimicry [30,31]. In PaTu8988 cells, both Akt inhibitor perifosine [41] and SAHA inhibited Sema-4D expression. Thus SAHA-exerted inhibitory effect against VM could also be associated Akt inhibition. More direct evidence is, however, needed to further support this hypothesis. In many cancer cells, over-expression or overactivation of growth factor receptors (i.e. EGFR, PDGFR) causes Akt hyper-activation [42]. Various inhibitors have been developed to target cell surface receptors or Akt (i.e. perifosine) for clinical use against cancers [42-45]. We found that SAHA significantly down-regulated EGFR and PDGFR expressions in PaTu8988 cells, which might be responsible for Akt inhibition. Once again, more direct evidence is still needed.

\section{Conclusions}

In summary, the above data demonstrated that SAHA possesses its anti-pancreatic cancer ability by inducing cell cycle arrest and cell apoptosis as well as suppressing tumor in vitro cell migration and VM. Akt inhibition might be associated with SAHA's inhibitory efficiency. Thus SAHA may be a potential anti-VM candidate for anti-pancreatic cancer therapy.

\section{Abbreviations}

SAHA: Suberoylanilide hydroxamic acid; VM: Vasculogenic mimicry; Sema-4D: Semaphorin-4D.

\section{Competing interests}

The authors have no conflict of interests. There were no financial competing interests.

\section{Authors' contributions}

$X X, L Z, L Y, Y P, Z C, Z Z$ and $Q Z$ carried out the experiments. $X X, L Y, L Z, C C$ and BY participated in the design of the study and performed the statistical analysis. XX, LY, CC and BY conceived of the study, and participated in its design and coordination and helped to draft the manuscript. All authors read and approved the final manuscript.

\section{Author details}

1Department of General Surgery, the Third Hospital affiliated to Soochow University, Changzhou City 213003, Jiangsu, China. ${ }^{2}$ Department of Neurology, the First Affiliated Hospital of Soochow University, Suzhou, China. ${ }^{3}$ Cyrus Tang Hematology Center, Soochow University, Suzhou 215123, Jiangsu, China. ${ }^{4}$ Jiangsu Key Laboratory of Translational Research and Therapy for Neuro-Psycho-Diseases and Institute of Neuroscience, Soochow University, Suzhou 215021, Jiangsu, China.

Received: 26 July 2013 Accepted: 16 May 2014

Published: 27 May 2014

\section{References}

1. Tinari N, De Tursi M, Grassadonia A, Zilli M, Stuppia L, lacobelli S, Natoli C: An epigenetic approach to pancreatic cancer treatment: the prospective role of histone deacetylase inhibitors. Curr Cancer Drug Targets 2012, 12:439-452.

2. Siegel R, DeSantis C, Virgo K, Stein K, Mariotto A, Smith T, Cooper D, Gansler T, Lerro C, Fedewa S, Lin C, Leach C, Cannady RS, Cho H, Scoppa S,
Hachey M, Kirch R, Jemal A, Ward E: Cancer treatment and survivorship statistics, 2012. CA Cancer J Clin 2012, 62:220-241.

3. Yeo CJ, Cameron JL: Improving results of pancreaticoduodenectomy for pancreatic cancer. World J Surg 1999, 23:907-912.

4. Assifi MM, Hines OJ: Anti-angiogenic agents in pancreatic cancer: a review. Anticancer Agents Med Chem 2011, 11:464-469.

5. Andersson R, Aho U, Nilsson BI, Peters GJ, Pastor-Anglada M, Rasch W, Sandvold ML: Gemcitabine chemoresistance in pancreatic cancer: molecular mechanisms and potential solutions. Scand J Gastroentero/ 2009, 44:782-786.

6. Weis SM, Cheresh DA: Tumor angiogenesis: molecular pathways and therapeutic targets. Nat Med 2011, 17:1359-1370.

7. Potente M, Gerhardt H, Carmeliet P: Basic and therapeutic aspects of angiogenesis. Cell 2011, 146:873-887

8. Folberg R, Hendrix MJ, Maniotis AJ: Vasculogenic mimicry and tumor angiogenesis. Am J Pathol 2000, 156:361-381.

9. Sun B, Qie S, Zhang S, Sun T, Zhao X, Gao S, Ni C, Wang X, Liu Y, Zhang L: Role and mechanism of vasculogenic mimicry in gastrointestinal stromal tumors. Hum Pathol 2008, 39:444-451.

10. Guzman G, Cotler SJ, Lin AY, Maniotis AJ, Folberg R: A pilot study of vasculogenic mimicry immunohistochemical expression in hepatocellular carcinoma. Arch Pathol Lab Med 2007, 131:1776-1781.

11. Seftor RE, Hess AR, Seftor EA, Kirschmann DA, Hardy KM, Margaryan NV, Hendrix MJ: Tumor cell vasculogenic mimicry: from controversy to therapeutic promise. Am J Pathol 2012, 181:1115-1125.

12. Favier J, Plouin PF, Corvol P, Gasc JM: Angiogenesis and vascular architecture in pheochromocytomas: distinctive traits in malignant tumors. Am J Pathol 2002, 161:1235-1246.

13. Lin SZ, Wei WT, Chen H, Chen KJ, Tong HF, Wang ZH, Ni ZL, Liu HB, Guo HC, Liu DL: Antitumor activity of emodin against pancreatic cancer depends on its dual role: promotion of apoptosis and suppression of angiogenesis. PLOS One 2012, 7:e42146

14. Qian DZ, Kato Y, Shabbeer S, Wei Y, Verheul HM, Salumbides B, Sanni T, Atadja P Pili R: Targeting tumor angiogenesis with histone deacetylase inhibitors: the hydroxamic acid derivative LBH589. Clin Cancer Res 2006, 12:634-642.

15. Kelly WK, O'Connor OA, Marks PA: Histone deacetylase inhibitors: from target to clinical trials. Expert Opin Investig Drugs 2002, 11:1695-1713.

16. Marks PA: Discovery and development of SAHA as an anticancer agent. Oncogene 2007, 26:1351-1356.

17. Kumagai T, Wakimoto N, Yin D, Gery S, Kawamata N, Takai N, Komatsu N, Chumakov A, Imai Y, Koeffler HP: Histone deacetylase inhibitor, suberoylanilide hydroxamic acid (Vorinostat, SAHA) profoundly inhibits the growth of human pancreatic cancer cells. Int J Cancer 2007, 121:656-665.

18. Ma X, Yang $Y$, Wang $Y, A n G$, Lv G: Small interfering RNA-directed knockdown of S100A4 decreases proliferation and invasiveness of osteosarcoma cells. Cancer Lett 2010, 299:171-181.

19. Zhou Q, Kiosses WB, Liu J, Schimmel P: Tumor endothelial cell tube formation model for determining anti-angiogenic activity of a tRNA synthetase cytokine. Methods 2008, 44:190-195.

20. Zhou Q, Kapoor M, Guo M, Belani R, Xu X, Kiosses WB, Hanan M, Park C, Armour E, Do MH, Nangle LA, Schimmel P, Yang XL: Orthogonal use of a human tRNA synthetase active site to achieve multifunctionality. Nat Struct Mol Biol 2010, 17:57-61.

21. Cao $C$, Huang $X$, Han $Y$, Wan $Y$, Birnbaumer L, Feng GS, Marshall J, Jiang M Chu WM: Galpha(i1) and Galpha(i3) are required for epidermal growth factor-mediated activation of the Akt-mTORC1 pathway. Sci Signal 2009, 2:ra17.

22. Cao C, Sun Y, Healey S, Bi Z, Hu G, Wan S, Kouttab N, Chu W, Wan Y: EGFR-mediated expression of aquaporin-3 is involved in human skin fibroblast migration. Biochem J 2006, 400:225-234.

23. Roy SK, Srivastava RK, Shankar S: Inhibition of PI3K/AKT and MAPK/ERK pathways causes activation of FOXO transcription factor, leading to cell cycle arrest and apoptosis in pancreatic cancer. J Mol Signal 2010, 5:10.

24. Parsons CM, Muilenburg D, Bowles TL, Virudachalam S, Bold RJ: The role of Akt activation in the response to chemotherapy in pancreatic cancer. Anticancer Res 2010, 30:3279-3289.

25. Furukawa T: Molecular targeting therapy for pancreatic cancer: current knowledge and perspectives from bench to bedside. J Gastroenterol 2008, 43:905-911.

26. Falasca M, Selvaggi F, Buus R, Sulpizio S, Edling CE: Targeting phosphoinositide 3-kinase pathways in pancreatic cancer-from molecular signalling to clinical trials. Anticancer Agents Med Chem 2011, 11:455-463. 
27. Azzariti A, Porcelli L, Gatti G, Nicolin A, Paradiso A: Synergic antiproliferative and antiangiogenic effects of EGFR and mTor inhibitors on pancreatic cancer cells. Biochem Pharmacol 2008, 75:1035-1044.

28. He L, Wu Y, Lin L, Wang J, Chen Y, Yi Z, Liu M, Pang X: Hispidulin, a small flavonoid molecule, suppresses the angiogenesis and growth of human pancreatic cancer by targeting vascular endothelial growth factor receptor 2-mediated PI3K/Akt/mTOR signaling pathway. Cancer Sci 2011, 102:219-225.

29. Garrido-Laguna I, Tan AC, Uson M, Angenendt M, Ma WW, Villaroel MC, Zhao M, Rajeshkumar NV, Jimeno A, Donehower R, lacobuzio-Donahue C, Barrett M, Rudek MA, Rubio-Viqueira B, Laheru D, Hidalgo M: Integrated preclinical and clinical development of mTOR inhibitors in pancreatic cancer. Br J Cancer 2010, 103:649-655.

30. Hess AR, Seftor EA, Seftor RE, Hendrix MJ: Phosphoinositide 3-kinase regulates membrane Type 1-matrix metalloproteinase (MMP) and MMP-2 activity during melanoma cell vasculogenic mimicry. Cancer Res 2003, 63:4757-4762.

31. Mei J, Gao Y, Zhang L, Cai X, Qian Z, Huang H, Huang W: VEGF-siRNA silencing induces apoptosis, inhibits proliferation and suppresses vasculogenic mimicry in osteosarcoma in vitro. Exp Oncol 2008, 30:29-34.

32. Lu JH, Shi ZF, Xu H: The mitochondrial cyclophilin D/p53 complexation mediates doxorubicin-induced non-apoptotic death of A549 lung cancer cells. Mol Cell Biochem 2014, 389:17-24.

33. Glantschnig H, Rodan GA, Reszka AA: Mapping of MST1 kinase sites of phosphorylation. Activation and autophosphorylation. J Biol Chem 2002, 277:42987-42996.

34. Chen B, Xu M, Zhang H, Wang JX, Zheng P, Gong L, Wu GJ, Dai T: Cisplatin-induced non-apoptotic death of pancreatic cancer cells requires mitochondrial cyclophilin-D-p53 signaling. Biochem Biophys Res Commun 2013, 437:526-531.

35. Chen MB, Wu XY, Tao GQ, Liu CY, Chen J, Wang LQ, Lu PH: Perifosine sensitizes curcumin-induced anti-colorectal cancer effects by targeting multiple signaling pathways both in vivo and in vitro. Int I Cancer 2012, 131:2487-2498.

36. Hu M, Xia M, Chen X, Lin Z, Xu Y, Ma Y, Su L: MicroRNA-141 regulates Smad interacting protein 1 (SIP1) and inhibits migration and invasion of colorectal cancer cells. Dig Dis Sci 2010, 55:2365-2372.

37. Fabre M, Garcia De Herreros A: Phorbol ester-induced scattering of HT-29 human intestinal cancer cells is associated with down-modulation of E-cadherin. J Cell Sci 1993, 106(Pt 2):513-521.

38. Spalletta G, Cravello L, Piras F, lorio M, Sancesario G, Marchi A, Caltagirone C, Cacciari C: Rapid-onset apathy may be the only clinical manifestation after dorsal striatum hemorrhagic lesion: a case report. Alzheimer Dis Assoc Disord 2013, 27:192-194.

39. Costello E, Neoptolemos JP: Pancreatic cancer in 2010: new insights for early intervention and detection. Nat Rev Gastroenterol Hepatol 2011, 8:71-73

40. Kim J, Kim YC, Fang C, Russell RC, Kim JH, Fan W, Liu R, Zhong Q, Guan KL: Differential regulation of distinct Vps34 complexes by AMPK in nutrient stress and autophagy. Cell 2013, 152:290-303.

41. Hideshima T, Catley L, Yasui H, Ishitsuka K, Raje N, Mitsiades C, Podar K, Munshi NC, Chauhan D, Richardson PG, Anderson KC: Perifosine, an oral bioactive novel alkylphospholipid, inhibits Akt and induces in vitro and in vivo cytotoxicity in human multiple myeloma cells. Blood 2006, 107:4053-4062.

42. Vivanco I, Sawyers CL: The phosphatidylinositol 3-Kinase AKT pathway in human cancer. Nat Rev Cancer 2002, 2:489-501.

43. Ravindran J, Prasad S, Aggarwal BB: Curcumin and cancer cells: how many ways can curry kill tumor cells selectively? AAPS J 2009, 11:495-510,

44. Duvoix A, Blasius R, Delhalle S, Schnekenburger M, Morceau F, Henry E, Dicato M, Diederich M: Chemopreventive and therapeutic effects of curcumin. Cancer Lett 2005, 223:181-190.

45. Liu J, Mao W, Ding B, Liang CS: ERKs/p53 signal transduction pathway is involved in doxorubicin-induced apoptosis in $\mathrm{H} 9 \mathrm{c} 2$ cells and cardiomyocytes. Am J Physiol Heart Circ Physiol 2008, 295:H1956-1965.

\section{doi:10.1186/1471-2407-14-373}

Cite this article as: Xu et al:: Suberoylanilide hydroxamic acid, an inhibitor of histone deacetylase, suppresses vasculogenic mimicry and proliferation of highly aggressive pancreatic cancer PaTu8988 cells. BMC Cancer 2014 14:373.

\section{Submit your next manuscript to BioMed Central and take full advantage of:}

- Convenient online submission

- Thorough peer review

- No space constraints or color figure charges

- Immediate publication on acceptance

- Inclusion in PubMed, CAS, Scopus and Google Scholar

- Research which is freely available for redistribution

Submit your manuscript at www.biomedcentral.com/submit
() Biomed Central 